Predictive modeling estimated that a $25 \%$ reduction in pNfLc, similar to that observed with ozanimod $0.92 \mathrm{mg}$, predicts an ARR (standard error [SE]) of $0.18-0.23(0.4)$, whereas a $13 \%$ reduction, similar to IFN, predicts an ARR (SE) of 0.29-0.37 (0.04).

Conclusion Our findings support pNfL-c as a biomarker for relapsing MS disease activity. Ozanimod caused greater dosedependent reductions in pNfL-c and ARR than IFN.

\section{BRADYCARDIA AS A RARE NEUROCARDIAC PRODROME TO LEUCINE-RICH GLIOMA INACTIVATED-1 ANTIBODY ENCEPHALITIS}

Sai Nagaratnam, Yun T Hwang, Elizabeth Reyneke. Neurology, Gosford Hospital, Gosford, NSW, Australia

\subsection{6/bmino-2021-ANZAN.68}

Introduction Leucine-rich glioma inactivated-1 antibody encephalitis has been associated with bradycardia as a neurocardiac prodrome. ${ }^{1}$ Concurrent occurrence of cardiac arrhythmia and faciobrachial dystonic seizures have not previously been reported.

Case A 73-year-old male presented with a 6 week history of frequent episodes of an unpleasant sensation associated with sinus bradycardia requiring pacemaker implantation. Episodes continued despite pacemaker. He was diagnosed with a seizure disorder and commenced on levetiracetam without response.

Subsequently, on video EEG, subtle facial grimace and upper limb tonicity were captured, in keeping with faciobrachial dystonic seizures without an EEG correlate. MRI Brain showed no radiological evidence of encephalitis. Serum limbic encephalitis panel confirmed LGI1 antibodies. Other autoimmune and paraneoplastic antibodies were negative. $\mathrm{He}$ was treated with a course of corticosteroids. Induction dose of intravenous immunoglobulin was prematurely terminated after one dose due to MRSA bacteraemia and tricuspid valve endocarditis, necessitating removal of the pacemaker with no recurrence of seizures or bradycardia at follow up without further treatment.

Conclusion This case illustrates a rare presentation of LGI-1 antibody encephalitis with complete remission following incomplete induction course of intravenous immunoglobulin and corticosteroids. Neurocardiac prodrome as episodic bradycardia or asystole may precede the onset of encephalitis by approximately 2 months. ${ }^{1} 2$ There is a good response to immunotherapy, however relapse is common. ${ }^{3}$ This case illustrates that clinically atypical presentations of cardiac arrhythmia may warrant neurological review and raises a possibility that early initiation of immunosuppressive therapy may significantly alter the disease course of LGI-1 antibody encephalitis.

\section{REFERENCES}

1. Naasan G, Irani SR, Bettcher BM, Geschwind MD, Gelfand JM. Episodic bradycardia as neurocardiac prodrome to voltage-gated potassium channel complex/leucine-rich, glioma inactivated 1 antibody encephalitis. JAMA Neurology 2014;71 (10):1300-4.

2. Nilsson AC, Blaabjerg M. More evidence of a neurocardiac prodrome in anti-LGI1 encephalitis. Journal of the Neurological Sciences 2015;357(1):310-1.

3. van Sonderen A, Thijs RD, Coenders EC, Jiskoot LC, Sanchez E, de Bruijn MAAM, et al. Anti-LGI1 encephalitis. Clinical Syndrome and Long-Term Follow-up 2016;87(14):1449-56 1,25hadi El-Wahsh, ${ }^{3}$ David Greenup, ${ }^{4}$ Gemma White, ${ }^{5,6}$ Michael J Fulham, ${ }^{3,7}$ Arun Aggarwal, ${ }^{1} \mathrm{G}$ Michael Halmagyi. ${ }^{1}$ Neurology, Royal Prince Alfred Hospital, Sydney, NSW, Australia; ${ }^{2}$ South Western Sydney Clinical School, University of New South Wales, Sydney, NSW, Australia; ${ }^{3}$ Department of Rehabilitation Medicine, Balmain Hospital, Sydney, NSW, Australia; ${ }^{4}$ Speech pathology department, Balmain Hospital, Sydney, NSW, Australia; ${ }^{5}$ Department of Molecular Imaging (PET and Nuclear Medicine), Royal Prince Alfred Hospital, Sydney, NSW, Australia; ${ }^{6}$ Sydney Medical School, University of Sydney, Sydney, NSW, Australia; ${ }^{7}$ Neurology, Concord Repatriation General Hospital, Sydney, NSW, Australia

\subsection{6/bmino-2021-ANZAN.69}

Objectives The role of subcortical structures in language function are still poorly understood. We aim to provide a putative mechanism for subcortical aphasia through a structural and functional imaging-based case discussion.

Methods We present a case of subcortical aphasia due to basal ganglia hypertensive haemorrhage and discuss serial MRI and PET imaging findings to elucidate the mechanism of profound language impairment in acute subcortical pathology.

Results A 71-year-old right-handed architect presented with acute onset global aphasia and right-sided hemiparesis. CT imaging showed a flame-shaped left-sided basal ganglia haemorrhage. MRI brain showed a left basal ganglia haemorrhage without ischaemic or haemorrhagic damage to the overlying fronto-parietal cortex. FDG-PET imaging showed profound left fronto-parietal cortex hypometabolism, as well as ipsilateral caudate, putamen, thalamic and pontine hypometabolism. MR tractography identified truncation of the arcuate fasciculus around the left angular gyrus as well as disconnection of the left fronto-parietal association fibres. Over 12 weeks of rehabilitation, the patient began to generate verbal output and was discharged home with ongoing word finding difficulties, nominal aphasia, and semantic paraphasias. Progress PET imaging revealed persistent hypometabolism in the aforementioned regions.

Conclusion We believe this is an important educational case for neurologists regarding the presentation of aphasia due to isolated subcortical lesions and raises some interesting hypotheses regarding a putative mechanism for subcortical aphasia due to dominant hemisphere cortical inactivation.

\section{HEMI-CORD INFARCTION FOLLOWING VERTEBRAL ARTERY DISSECTION IN A PATIENT WITH CONGENITAL HYPOPLASTIC VERTEBRAL ARTERY: A CASE REPORT}

${ }^{1}$ Alanna Rottler, ${ }^{1,2}$ Yew Li (Michelle) Dang, 1,3Wai Foong Hooi, ${ }^{4}$ David A Burrows, ${ }^{4,5}$ Hong Kuan Kok, ${ }^{1,6}$ Douglas E Crompton. 'Department of Neurology, Northern Health, Epping, VIC, Australia; ${ }^{2}$ Department of Neurology, Eastern Health, Box Hill, VIC, Australia; ${ }^{3}$ Department of Neurology, Austin Health, Heidelberg, VIC, Australia; ${ }^{4}$ Department of Radiology, Northern Health, Epping, VIC, Australia; ${ }^{5}$ School of Medicine, Faculty of Health, Deakin University, Burwood, VIC, Australia; ${ }^{6}$ Department of Medicine, Melbourne University, Northern Health, Epping, VIC, Australia

\subsection{6/bmjno-2021-ANZAN.70}

Background Whilst often causing posterior circulation strokes, vertebral artery dissections may also, more rarely, cause spinal cord infarction. ${ }^{1}$ This is the case report of a 39 -year-old female with a right-sided high cervical hemi-cord infarction caused by vertebral artery dissection of a hypoplastic right vertebral artery. 
Presentation A 39-year-old female with a history of migraines, presented with acute onset right neck pain, headache and right-sided paraesthesia of the arm and leg after rapid rotation of the neck to the left. Due to a headache similar to her usual migraine, the patient took Rizatriptan prior to presentation. Neurological examination revealed findings, including right-sided upper and lower limb paraesthesia, weakness and dysmetria, consistent with right hemi-cord infarction. CT angiogram of the neck and brain revealed a small calibre right vertebral artery of unclear aetiology. Subsequent MRA revealed a hypoplastic right vertebral artery with dissection causing a high cervical right hemi-cord infarction. The patient's right sided paraesthesia and weakness slowly improved over three months with medical therapy and rehabilitation, however some deficits remain, which affect her quality of life.

Conclusion This unusual case is a poignant reminder to carefully consider alternative diagnoses that may mimic migraines, especially when neurological signs and symptoms are present. Such differential diagnoses, such as vertebral artery dissection and spinal cord infarction, are of particular importance to consider, even in young patients without any risk factors, given that they can cause significant disability which may impact on quality of life. ${ }^{2}$

\section{REFERENCES}

1. Hsu J, Cheng M, Liao M, Hsu H, Weng Y, Chang K, et al. The etiologies and prognosis associated with spinal cord infarction. Annals of Clinical and Translational Neurology 2019;6(8):1456-1464.

2. Hsu C, Cheng C, Lee J, Lee M, Huang Y, Wu C, et al. Clinical features and outcomes of spinal cord infarction following vertebral artery dissection: a systematic review of the literature. Neurological Research 2013;35(7):676-683.

\section{DOUBLE TROUBLE: PAPILLOEDEMA SECONDARY TO IDIOPATHIC INTRACRANIAL HYPERTENSION AND APLASTIC ANAEMIA}

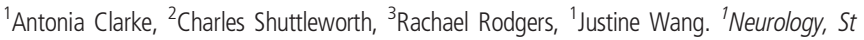
George Hospital, Kogarah, NSW, Australia; ${ }^{2}$ Haematology, St George Hospital, Kogarah, NSW, Australia; ${ }^{3}$ Obstetrics and Gynaecology, Royal Hospital for Women, Randwick, NSW, Australia

\subsection{6/bmjno-2021-ANZAN.71}

Objective We report the case of a 34-year-old female diagnosed concurrently with idiopathic intracranial hypertension (IIH) and aplastic anaemia.

Case A 34-year-old female with recent weight gain presented with headache and fatigue. Clinical examination revealed conjunctival pallor and occasional bruising, with fundoscopy and optic coherence tomography demonstrating bilateral papilloedema (grade III). There were enlarged blind spots bilaterally. The cerebrospinal fluid (CSF) opening pressure was greater than $30 \mathrm{cmH}_{2} \mathrm{O}$. An MRI brain was normal and there was no venous sinus thrombosis. A diagnosis of IIH was made, and she was treated with therapeutic removal of CSF, acetazolamide and weight loss strategies. Concurrently, a bone marrow biopsy to investigate profound panyctopaenia was consistent with aplastic anaemia. The patient had worsening IIH features during fertility preservation treatment in preparation for stem cell transplant. At five months, there was complete resolution of subretinal fluid and clinical papilloedema. Anti-thymocyte globulin and cyclosporine treatment was subsequently commenced.
Conclusion Previous case reports have emphasised the interplay between the pathophysiology of anaemia and $\mathrm{IIH},{ }^{1}$ with treatment of aplastic anaemia contributing to resolution of $\mathrm{IIH}^{2,3}$ We believe this is the first reported case of concomitant IIH and aplastic anaemia with resolution of papilloedema prior to treatment of anaemia. We also highlight the challenges of managing IIH during fertility and cyclosporine treatment.

\section{REFERENCES}

1. Biossue $\mathrm{V}$, et al. Anaemia and papilledema. American Journal of Ophthalmology 2003;135(4):437.

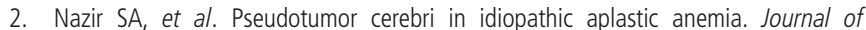
AAPOS 2003;7(1):71.

3. Lilley ER, Bruggers CS, Pollock SC. Papilledema in a patient with aplastic anemia. Arch Ophthalmol 1990;108:1674-5.

\section{2 IMPACT OF TELEHEALTH ON MULTIPLE SCLEROSIS (MS) OUTPATIENT CLINICS DURING THE COVID-19 PANDEMIC}

Vivien Li, Ai-Lan Nguyen, Izanne Roos, Katherine Buzzard, Chris Dwyer, Mark Marriott, Mastura Monif, Charles Malpas, Stefanie Roberts, Lisa Taylor, Elizabeth Carle, Nicola Taylor, Kelsey Tunnell, Trevor Kilpatrick, Tomas Kalincik. Neurology, Royal Melbourne Hospital, Parkville, VIC, Australia

\subsection{6/bmjno-2021-ANZAN.72}

\section{Objectives}

1. Characterise telehealth use in MS clinics during the COVID19 pandemic.

2. Assess patient and clinician attitudes towards telehealth.

3. Compare telehealth-based and physical EDSS obtained during period of telehealth implementation.

Methods Clinic records from Mar-Dec 2020 were reviewed. Patients and clinicians completed questionnaires about experiences using Telehealth. The iMed database was searched for EDSS recorded via face-to-face and telehealth appointments during and compared to face-to-face EDSS preceding and following the study period. T-test and Chi-square test were used for between-group comparisons.

Results 2023 appointments (27\% face-to-face, $35 \%$ video, $37 \%$ telephone) were conducted. New referrals were predominantly face-to-face (66\%).

$89 \%$ of patients were satisfied with telehealth. $58 \%$ felt they were as good as face-to-face visits, whilst only $11 \%$ of clinicians agreed. Many patients favoured a hybrid model. Safety during the COVID-19 pandemic was important to both groups.

EDSS increase from the preceding visit was recorded in a significantly higher proportion of face-to-face than telehealth appointments $(\mathrm{p}=0.027)$, with the increase driven by patients with baseline EDSS $\leq 4.0$. Amongst patients with EDSS increases, similar numbers of suspected relapses were seen via both modalities. Absolute increase in EDSS was also significantly greater amongst patients seen face-to-face $(\mathrm{p}<0.0001)$. There was no significant difference in EDSS change at subsequent follow-up in patients with consecutive face-to-face versus intervening telehealth appointments.

Conclusion Patient satisfaction with telehealth was high, whilst clinicians preferred face-to-face consultations. EDSS increase was more frequently recorded via face-to-face than telehealth 